\title{
Gossypol Promotes Bone Formation in Ovariectomy-Induced Osteoporosis through Regulating Cell Apoptosis
}

\author{
Jinqian Liang, ${ }^{1}$ Chong Chen, ${ }^{1}$ Hongzhe Liu, ${ }^{2}$ Xiangyang Liu, ${ }^{2}$ Zheng Li, ${ }^{1}$ \\ Jianhua Hu $\mathbb{D}^{1},{ }^{1}$ and Hong Zhao $\mathbb{D D}^{1}$ \\ ${ }^{1}$ Department of Orthorpaedic Surgery, Peking Union Medical College Hospital, Beijing 100730, China \\ ${ }^{2}$ Department of Orthopedics, People's Hospital of Hunan Province, Hunan 410005, China \\ Correspondence should be addressed to Jianhua Hu; hujianhuapumch@126.com and Hong Zhao; hzhao1234@yeah.net
}

Received 30 August 2018; Accepted 5 November 2018; Published 13 December 2018

Academic Editor: Gjumrakch Aliev

Copyright (C) 2018 Jinqian Liang et al. This is an open access article distributed under the Creative Commons Attribution License, which permits unrestricted use, distribution, and reproduction in any medium, provided the original work is properly cited.

Osteoporosis is among the most common forms of age-related diseases, especially for females, which has been a grave public health problem. Drug therapies have shown promising outcomes to promote bone formation and bone density. This study identified a novel potential drug, gossypol, for the treatment of osteoporosis. Treatments of ovariectomy-induced osteoporosis mice with gossypol significantly increased serum osteocalcin and osteoprotegerin (OPG) levels; meanwhile they decreased serum RANKL levels. Microcomputed tomography (microCT) analysis showed that treatment of gossypol improved bone density and strength and decreased bone postyield displacement for both medullar and cortical bones. In vitro experiments also showed that gossypol increased cell viability in a time- and dose-dependent manner. Furthermore, incubation of the osteoblast MC3T3-E1 cells with gossypol inhibited cell apoptosis through intrinsic apoptotic pathway as evidenced by the Annexin V/PI assay, TUNEL assay, biochemical analysis, and western blot assays. Moreover, the classical Wnt/ $\beta$-catenin signaling pathway was found to be regulated by gossypol treatments. Inhibition of Wnt/ $\beta$-catenin signaling reversed the prevention effects of gossypol in osteoporosis. Our findings provided novel clues for the treatment of osteoporosis in clinic.

\section{Introduction}

Osteoporosis is one of the most common causes for a broken bone among the elderly [1], which may result from lower bone mass and greater bone loss [2]. Osteoporosis may occur due to aging and a variety of diseases or treatments including alcoholism, hyperthyroidism, kidney failure, and surgical removal of the ovaries $[3,4]$. The underlying mechanism of all kinds of osteoporosis is an imbalance between bone resorption and bone formation, resulting from mesenchymal stem cells (MSC) biasing from osteoblast and towards the marrow adipocyte lineage $[5,6]$. Of note, estrogen strongly determines the rate of bone resorption, lack of which increases bone resorption and decreases the deposition of new bones that take place in weight-bearing bones [7]. In addition to hormones, calcium metabolism appears to be one of the most important factors in bone turnover. Deficiency of calcium and vitamin D may lead to impaired bone deposition $[8,9]$.

Lifestyle prevention and nutrition supplement with calcium and vitamin D are two main solutions to relieve osteoporosis [10]. Besides, medication intervene is another effective way, such as bisphosphonates, which decreases the risk of future fractures in individuals who have already sustained a fracture after osteoporosis [11]. However, for those with osteoporosis but not with a fracture evidence, risedronate or etidronate is not supportive for a reduction in fracture risk [12]. Fluoride supplement is not effective enough for postmenopausal osteoporosis since it increases bone density but does not decrease the risk of fractures [13]. Thus, there is still a long way to go to cure osteoporosis.

Gossypol is a natural product derived from the genus Gossypium, which is a phenolic aldehyde and acts as an inhibitor of various dehydrogenase enzymes [14]. Besides its 
putative contraceptive function, gossypol is a well-known antimalarial and a potential anticancer drug [15]. Recently, Saleh et al. reported the critical role of gossypol in cell inflammation and oxidative stress [16]. Chen et al. demonstrated gossypol could stimulate the opening of a $\mathrm{Ca}^{2+}-$ and $\mathrm{Na}^{+}$- permeable but $\mathrm{Ni}^{2+}$ - and $\mathrm{Co}^{2+}$ - impermeable pore in endothelial cells [17], hinting that gossypol may be related to the progression of osteoporosis. However, whether it is effective for bone formation during the development of osteoporosis has never been discovered. Herein, we studied the role of gossypol in osteoporosis induced by ovariectomy in vivo and in vitro. Histology, microCT and blood index were included and mouse MC3T3-E1 cell line was used for functional analysis.

\section{Materials and Methods}

2.1. Animals. This study was approved by the Ethic Committee of Department of Orthopedic, People's Hospital of Hunan Province. A total of 20 C57BL/6 mice (age: 8 weeks, male) were purchased from Model Animal Research Center of Nanjing University. All of the mice were housed under standard conditions of 12h: 12h dark/light cycles. Gossypol was purchased from APExBIO Co. (Catalog number: N2135, NY, USA) and dissolved in DMSO with a concentration of $15 \mathrm{mg} / \mathrm{ml}$. All mice underwent oophorectomy and then were randomly divided into two groups: control group (i.p. injection with DMSO) and gossypol (i.p. injection with gossypol with a dose of $15 \mathrm{mg} / \mathrm{kg}$ ). The injection was continued every day for four weeks. Afterwards, mice were sacrificed after anesthesia with $7 \%$ pentobarbital sodium and the thighbone of each mouse was harvested.

2.2. Cell Culture. MC3T3-E1 cell line deprived from neonate C57BL/6 mice was purchased from American Type Culture Collection (CRL-2593, Massachusetts, USA) and cultured in DMEM (Gibco, NY, USA) supplemented with $10 \%$ fetal bovine serum (FBS, Gibco). Wnt/ $\beta$-catenin signaling pathway inhibitor, ICG-001, was purchased from Selleck (Shanghai, China) and dissolved in DMSO with a concentration of $50 \mathrm{mM}$. Cells were treated with gossypol with a concentration of $20 \mathrm{~nm}$ for $24 \mathrm{~h}$, unless otherwise stated.

2.3. Histology. The thighbone from each mouse was fixed in $10 \%$ paraformaldehyde and decalcified with a Sigma-Aldrich decalcifying reagent. After dehydrating for 18 hours, the tissues were embedded with paraffin and cut into $5 \mu \mathrm{m}$ slices for hematoxylin and eosin (H\&E) staining. Five random fields were selected and photographed under a Nikon (Japan) light microscope to calculate bone formation and osteoporosis.

2.4. Elisa Assays. Blood samples from each mouse were collected for biochemical analyses. The serum osteocalcin levels were assessed with a Life Technology Enzyme-linked immunosorbent assay kit (Elisa, CA,USA) according to the manufactures' instructions. Serum RANKL and osteoprotegerin (OPG) levels were determined with Elisa kits from R\&D system (Minneapolis, MN, USA) as per the protocols.
2.5. MicroCT Analysis. A microcomputed tomography (microCT) system (Bassersdorf, Switzerland) and the corresponding analysis software were applied for the microCT analysis. Briefly, the samples were harvested and $\mathrm{X}$-rays previewing was performed using a $1024 \times 256$ element CCD array, which was fixed with a Compaq/hp $\alpha$-Station operating in an open VMS environment. Samples were scanned on femurs with medium resolutions. After each scanning, the system got an image dataset by 1024 twodimensional axial slices at the midshaft region of femurs. It took about 5 minutes for each bone sample.

2.6. Induction of MC3T3-E1 Cells Differentiation into Osteoblasts. To differentiate into osteoblasts, MC3T3-E1 cells were seeded on 24 -well plates at a density of $6.5 \times 10^{4}$ cells and cultured in $\alpha$-MEM supplemented with $10 \mathrm{mM}$ $\beta$-glycerol phosphate, $50 \mu \mathrm{g} / \mathrm{ml}$ ascorbic acid, and $10 \mu \mathrm{M}$ dexamethasone for 21 days. The media of cultured cells were replaced every 3 days.

2.7. Cell Viability Assays. Cell viability was assessed with the methylthiazoletetrazolium (MTT) assay. Briefly, cells were treated with gossypol for different dose $(0,1,5,10$, and $20 \mathrm{nM})$ and various time periods $(0,6,12,18$, and $24 \mathrm{~h})$. Afterwards, cells were trypsinized and reseeded in triplicate in a 96-well plate at an initial density of 4,000 cells/well. Then, cells were added with $10 \mu \mathrm{l}$ of MTT solution $(5 \mathrm{mg} / \mathrm{mL}$ ) per well and incubated for $2 \mathrm{~h}$ at $37^{\circ} \mathrm{C}$; the absorbance of each well was recorded at $570 \mathrm{~nm}$. Cell viability was calculated as the cell number ratio of experimental groups to control cells.

2.8. Apoptotic Analysis. The Annexin V/ PI assay was conducted as per the manufacturer's instructions (Invitrogen, NY, USA). Briefly, MC3T3-E1-differentiated osteoblast cells were treated with gossypol with a concentration of $20 \mathrm{nM}$ for 24 hours. Afterwards, cells were washed with prechilled PBS, trypsinized with trypsin for $1 \mathrm{~min}$, and resuspended in $100 \mu \mathrm{l}$ of binding buffer supplemented with $2.5 \mu$ l FITC conjugated Annexin V and $1 \mu \mathrm{l}$ PI $(100 \mu \mathrm{g} / \mathrm{ml})$. Then, cells were shaken at room temperature for $15 \mathrm{~min}$ in a void of light. A total of 10 , 000 cells were collected and assessed by flow cytometry (BD Biosciences).

2.9. Terminal Deoxynucleotidyl Transferase dUTP Nick End Labeling (TUNEL) Assays. The TUNEL assays were performed according to the protocols (Vazyme, Nanjing, China). Cells were stimulated with gossypol for 24 hours and then washed with ice-cold PBS. After incubation with lysis buffer for $10 \mathrm{~min}$ on ice, the cell pellet was collected with lowspeed centrifugation $\left(1000 \mathrm{~g}, 5 \mathrm{~min}, 4^{\circ} \mathrm{C}\right)$. Slides with adherent cells were covered with 50 ul terminal deoxynucleotidyl transferase reaction mixture for $60 \mathrm{~min}$ at $37^{\circ} \mathrm{C}$ in darkness. After being stopped with SSC for $15 \mathrm{~min}$, nuclei were stained and visualized with DAPI staining. Five random fields were photographed with the Nikon light microscope.

2.10. Relative Caspase Activities. The activities of caspase-3, caspase- 8 , and caspase- 9 were assessed with caspase activity 
TABle 1: Primers used in this study.

\begin{tabular}{lll}
\hline Gene & Forward & Reverse \\
\hline Osteocalcin & ACACTCCTCGCCCTATTG & GATGTGGTCAGCCAACTC \\
Osterix & ACACTCCTCGCCCTATTG & TAGAAGGAGCAAGGGGACAGAA \\
Runx2 & TCTGGAAAAAAAAGGAGGGACTATG & GGTGCTCGGATCCCAAAAGAA \\
ODF & CACCTGGTTGCTGACTAATTGAGA & CTTGCTGTCCGACCAAATA \\
OPG & AACGGCAACACAGCTCACAAGAAC & TGCTCGAAGGTGAGGTTAGCATGT \\
Wnt & CGATGGTGGGGTATTGTGAAC & CCGGATTTTGGCGTATCAGAC \\
AKP & CCAACTCTTTTGTGCCAGAGA & GGCTACATTGGTGTTGAGCTTTT \\
GSK-3 $\beta$ & ATGGCAGCAAGGTAACCACAG & TCTCGGTTCTTAAATCGCTTGTC \\
TGF $\beta 1$ & CCACCTGCAAGACCATCGAC $1 A 1$ & CTGGCGAGCCTTAGTTTGGAC \\
$\beta$-catenin & CACCAATCACCTGCGGTACAGAA & CAGATCACGTCATCGCACAAC \\
GAPDH & AAAGCGGCTGTTAGTCACTGG & CGAGTCATTGCATACTGTCCAT \\
\hline
\end{tabular}

kits (Beyotime, Nantong, China). Briefly, cells were treated with gossypol for 24 hours and then collected by low-speed centrifugation $\left(1000 \mathrm{~g}, 5 \mathrm{~min}, 4^{\circ} \mathrm{C}\right)$. A total of $100 \mu \mathrm{g}$ proteins from each sample were added to a 96-well plate and mixed with an aliquot of $80 \mu \mathrm{l}$ reaction buffer as well as caspase-3, caspase- 8 , and caspase- 9 substrates $(2 \mathrm{mM})$. After incubation at $37^{\circ} \mathrm{C}$ for $4 \mathrm{~h}$, caspase activities were determined by the microplate reader at an absorbance of $450 \mathrm{~nm}$ and the data were normalized to the control cells.

2.11. Western Blot Analysis. Osteoblast cells were treated with gossypol with a concentration of $20 \mathrm{nM}$ for 24 hours. Afterwards, cells were harvested with lysis buffer (NP-40, Beyotime, Nantong, China) and a total of $10 \mu \mathrm{g}$ proteins were loaded up to a $10 \%$ SDS-PAGE gel. After being transferred to a nitrocellulose membrane, the proteins were blocked with 5\% skim milk in TBST at room temperature for 1 hour and incubated with primary antibodies at $4^{\circ} \mathrm{C}$ overnight. The primary antibodies against osteocalcin (ab13420), osterix (ab209484), Runx2 (ab23981), Wnt1 (ab15251), $\beta$-Catenin (ab16051), and COL1A1 (ab90395) were purchased from Abcam (Cambridge, USA) and diluted into 1:1000 for use. The primary antibodies against ODF (sc-390152), AKP (sc-271431), GAPDH (sc47724), and secondary antibodies (1:5000) were commercially from Santa Cruz Biotechnology (Santa Cruz, CA, USA). The primary antibody against TGF $\beta 1$ was from Proteintech Inc. (21898-1-AP, 1:1000) (Wuhan, China).

2.12. Real-Time Polymerase Chain Reaction (RT-PCR). Total RNAs from cells were extracted with a standard Trizol solution (Thermo Scientific, NY, USA). RNAs were quantified by Nanodrop 2000 by collecting the OD260 and OD280 absorbance and an amount of 1000ng RNA was reversely transcribed into cDNA with Prime Script TM Master Mix (Takara, Japan). qRT-PCR was performed with SYBR Premix EX Taq TM II (Takara) on the real-time PCR detection system ABI7500 (Thermo Fisher Scientific, CA, USA). GAPDH was included as an inner control and gene mRNA expression was calculated by $2^{-\Delta \Delta C t}$ method. The qPCR protocol was shown as follows: initial denaturation at $95^{\circ} \mathrm{C}$ for $5 \mathrm{~min}$, followed by
45 repeats of a three-step cycling program consisting of $10 \mathrm{sec}$ at $95^{\circ} \mathrm{C}$ (denaturation), $10 \mathrm{~s}$ at $60^{\circ} \mathrm{C}$ (primer annealing), and 10 $\mathrm{sec}$ at $72^{\circ} \mathrm{C}$ (elongation) and a final extension step for $10 \mathrm{~min}$ at $72^{\circ} \mathrm{C}$. The primers used were listed in Table 1.

2.13. Statistical Analysis. GraphPad Prism (GraphPad Software, La Jolla, CA, USA) software was included for statistical analysis. Data were shown as mean \pm standard deviation (SD). The two-tailed Student's $t$-test was used to compare means between two groups and one-way analysis of variance (ANOVA) was used for comparisons among multiple groups ( $\geq 3$ groups), followed by a LSD post hoc test. Chi-square or Fisher's exact test was used to compare proportion differences of categorical variables. Differences were considered statistically significant when a two-sided $p$ value was less than 0.05 .

\section{Results}

3.1. Gossypol Promotes Bone Formation in OvariectomyInduced Osteoporosis In Vivo. To evaluate the effects of gossypol on bone formation, we first performed histological analysis with ovariectomy-induced osteoporosis mice. As shown in Figure 1(a), gossypol-injected mice showed relieved osteoporosis as characterized by a remarkable increase in the trabeculae of epiphyseal (red arrows) and metaphyseal regions (black arrows) when compared with the control mice. The increased thickness of cortical bone was also observed in the metaphyseal region in the gossypol-treated group (data not shown). Furthermore, we also analyzed several serum chemicals with Elisa methods in both groups. It was shown that the serum osteocalcin levels in gossypol-injected mice were significantly higher than the control counterpart (Figure 1(b)). Furthermore, the serum OPG levels in the experimental mice were increased to 2-fold of the control group (Figure 1(c)) and the serum RANKL levels were decreased by approximate $50 \%$ (Figure $1(\mathrm{~d})$ ), causing the ratio of RANKL to OPG to drop to $25 \%$ in the gossypol-injected mice in comparison with the control group (Figure 1(e)).

Next, microCT scanning was performed and found that, as compared with vehicle-treated osteoporosis mice, gossypol-injected mice had higher medullar and cortical 


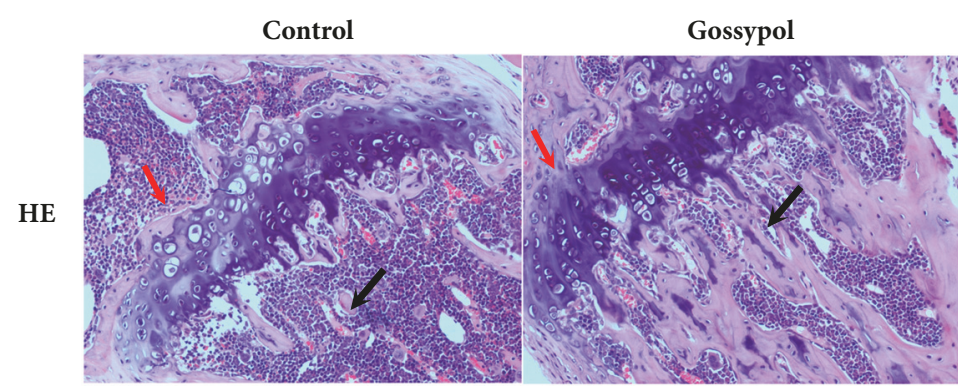

(a)

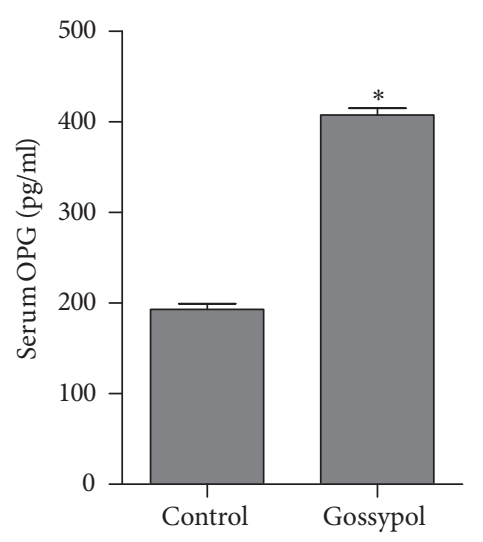

(c)

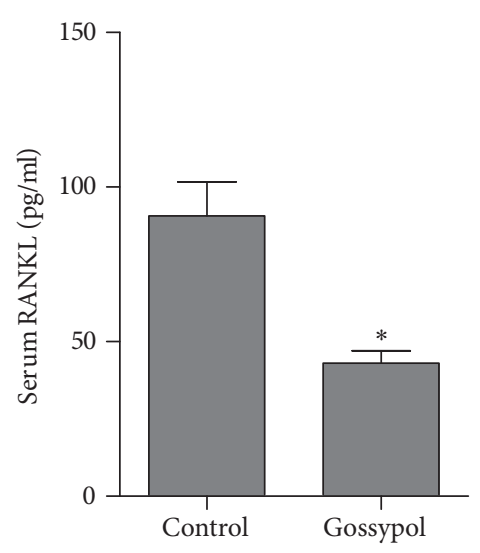

(d)

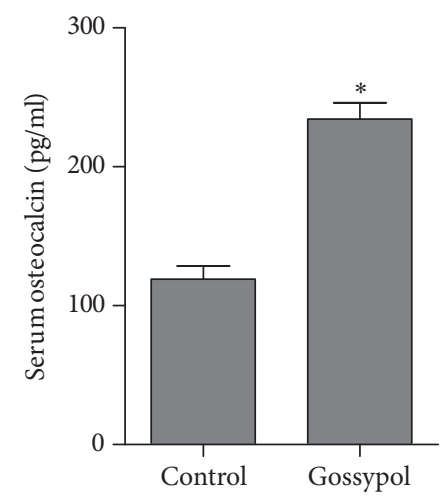

(b)

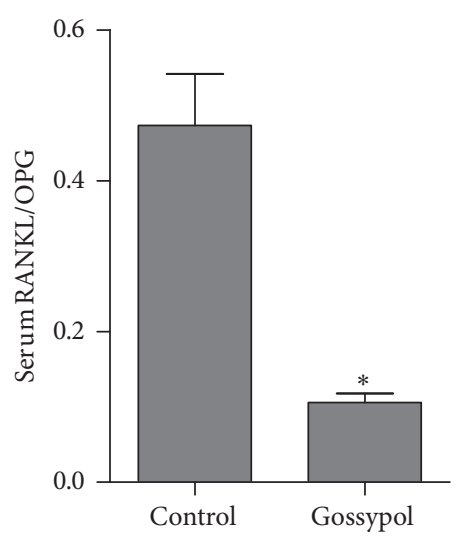

(e)

FIGURE 1: Gossypol promotes bone formation in ovariectomy-induced osteoporosis in vivo. (a) Mice underwent ovariectomy in the presence or absence of gossypol injection and the thighbone of each mouse was collected for H\&E staining. Red arrow means trabeculae of epiphyseal and black arrow means trabeculae in metaphyseal regions. (b) Serum osteocalcin level of each mouse in control and gossypol injection groups was determined with Elisa methods. (c) Serum OPG levels were assessed with Elisa methods for the mice injected with solvent or gossypol. (d) Serum RANKL levels of mice from two groups were measured with Elisa methods. (e) Serum RANKL/OPG levels were assessed by the ratio of serum RANKL and serum OPG levels. Each experiment was repeated for three times. Data were presented as mean \pm SEM. $* P<0.05$ versus control.

bone density (Figure 2(a)). Furthermore, the bone density of control mice was approximately $400 \mathrm{mg} / \mathrm{cm}^{3}$, which was significantly lower than that in the gossypol-treated mice $\left(600 \mathrm{mg} / \mathrm{cm}^{3}\right)$ (Figure 2(b)). It was further shown that gossypol treatment rescued ovariectomy-induced bone strength loss by almost $50 \%$ as compared to the control group (Figure 2(c)). Moreover, the postyield displacement of gossypol-injected mice was also dropped to $50 \%$ of the control counterparts (Figure 2(d)). These data together with Figure 1 suggested that gossypol promotes bone formation in ovariectomy-induced osteoporosis mice.

3.2. Gossypol Promotes Cell Viability in Osteoblast Cell Line MC3T3-E1 In Vitro. To identify the specific role of gossypol in bone formation, we further examined the cell viabilities of osteoblast cell line MC3T3-E1 upon gossypol treatment. After differentiation, MC3T3-E1 cells were treated with gossypol for various time periods. It was shown that the cell viability was increased at the time checkpoint $12 \mathrm{~h}$ and the further upregulated with the prolonged time periods $18 \mathrm{~h}$ and $24 \mathrm{~h}$
(Figure 3(a)). Moreover, higher doses of gossypol increased cell viability in a dose-dependent manner as compared with control cells (Figure 3(b)). These results showed gossypol promoted cell viability in osteoblasts in a time- and dosedependent manner.

3.3. Gossypol Inhibits Cell Apoptosis in Osteoblast Cells In Vitro. Next, we examined the effects of gossypol on cell apoptosis. To this end, MTT assays were performed, which showed that the apoptotic rate of MC3T3-E1 cells in gossypoltreated group was notably decreased by $8 \%$ compared with the control cells (Figure 4(a)). TUNEL assay also revealed that the apoptotic cells were rescued by gossypol stimulation in vitro (Figure $4(\mathrm{~b})$ ). Moreover, we primitively checked the two signaling pathways of apoptosis: intrinsic and extrinsic pathways with RT-PCR and western blot assays. It was shown in Figure 4(c) that the relative activities of caspase-3 and caspase-9, which were both involved in the intrinsic pathway, were remarkably decreased by more than $50 \%$ in gossypoltreated cells, while that of caspase-8, a critical caspase 


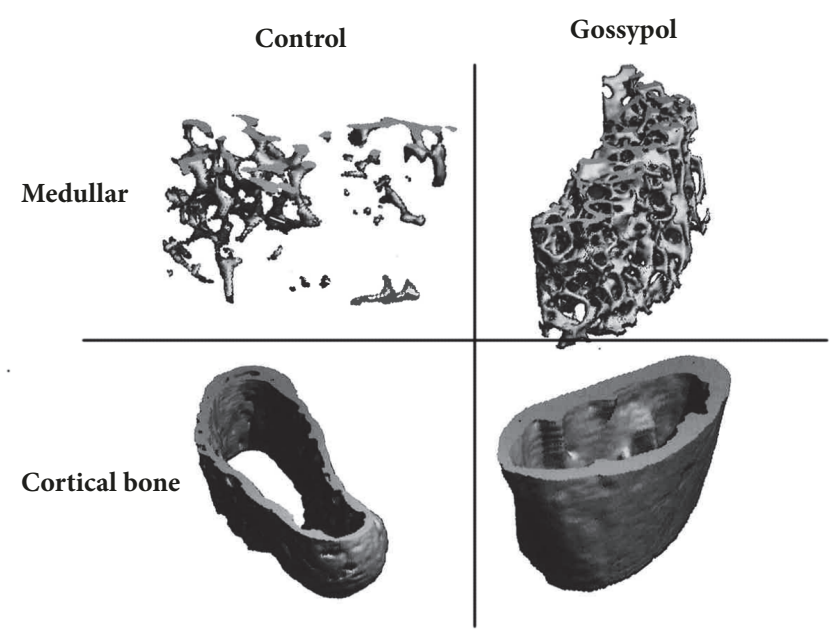

(a)

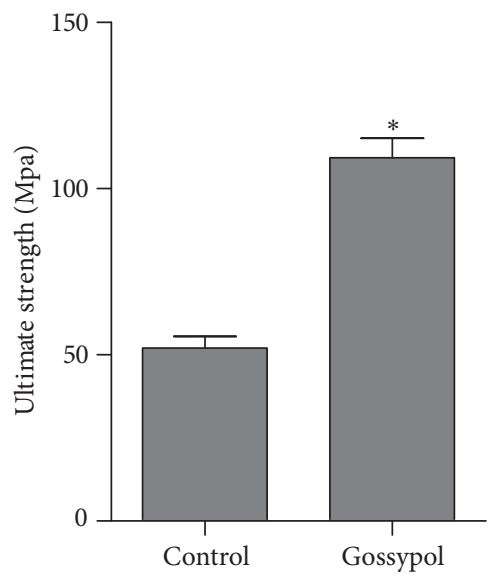

(c)

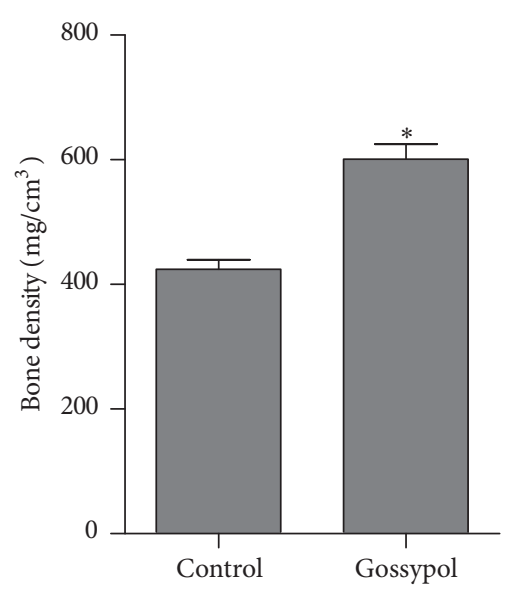

(b)

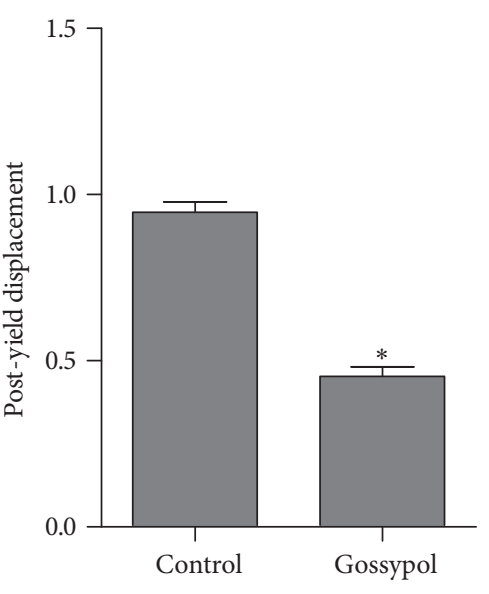

(d)

FIGURE 2: Gossypol increased bone strength after ovariectomy. (a) MicroCT was used to scan the thighbone of each mouse in control and gossypol group. (b) Bone density of each mouse was assessed in control and gossypol group. (c) Ultimate strength of each mouse was determined in control and gossypol group. (d) Postyield displacement of each mouse was measured in control and gossypol group. Each experiment was repeated for three times. Data were presented as mean \pm SEM. $* P<0.05$ versus control.

involved in extrinsic pathway, remained stable. Similarly, gossypol treatment led to the protein levels of caspase- 3 and caspase-9 dropping significantly, while causing no effects to that of caspase-8. All of these data suggested gossypol inhibited cell apoptosis in MC3T3-E1 cells through intrinsic apoptotic pathway.

\subsection{Gossypol Promotes Bone Formation through Wnt/ $\beta$ -} Catenin Signaling Pathway. To elucidate the detailed mechanism of how gossypol regulates cell apoptosis, several signaling pathways were examined, among which $\mathrm{Wnt} / \beta$-catenin showed the best response to gossypol treatments. Western blot analysis showed that gossypol treatment significantly upregulated the protein levels of Wnt, $\beta$-catenin, GSK-3 $\beta$, and TGF $\beta 1$ (Figure 5(a)). Furthermore, the mRNA levels of Wnt, $\beta$-catenin, GSK-3 $\beta$, and TGF $\beta 1$ in gossypol-treated group were also increased by more than 2 -fold compared to those in the control cells (Figure 5(b)). These data showed gossypol promoted Wnt $/ \beta$-catenin signaling pathway in MC3T3-E1 osteoblast cells.

Next, we intervened the Wnt/ $\beta$-catenin signaling pathway with its inhibitor ICG-001 and examined the expression of related genes. As shown in Figure 6(a), gossypol treatment increased the mRNA levels of bone formation related genes, such as osteocalcin, osterix, Runx2, ODF, OPG, AKP, TGF $\beta 1$, and COL1A1 in comparison with the control cells. Likewise, all of these proteins were upregulated by gossypol treatment and reversed after coincubation with Wnt $/ \beta$-catenin pathway inhibitor ICG-001 (Figure 6(b)). All of these observations demonstrated that gossypol promoted bone formation through activating $\mathrm{Wnt} / \beta$-catenin pathway.

\section{Discussion}

Similar to the senile osteoporosis that is also caused due to the lack of estrogen, ovariectomy could lead to the imbalance 


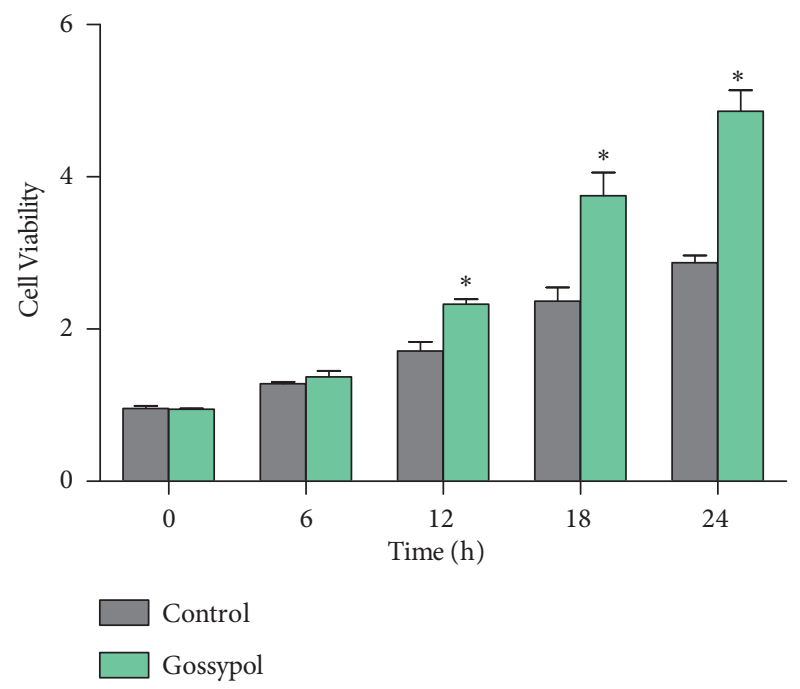

(a)

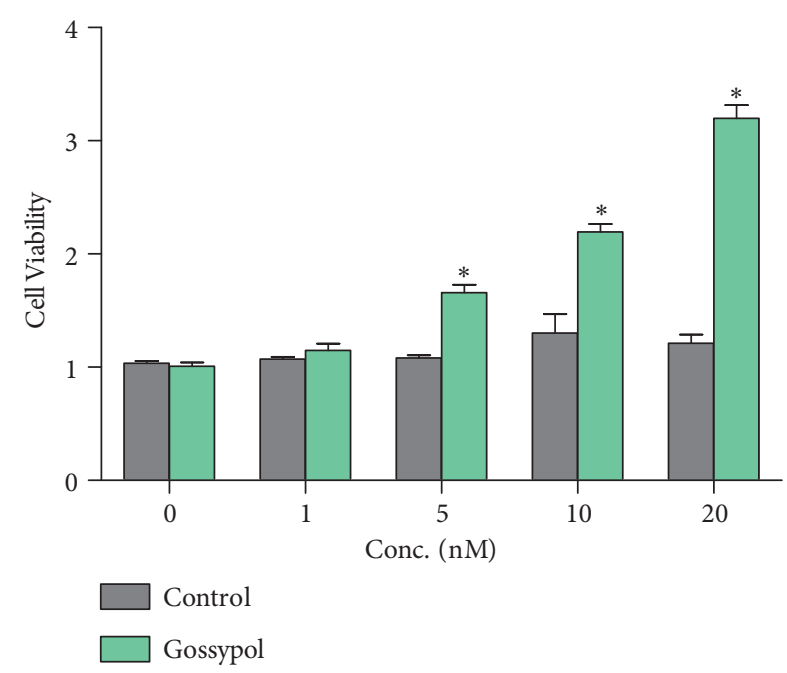

(b)

FIGURE 3: Gossypol promotes cell viability in osteoblast cell line MC3T3-E1 in vitro. (a) Cell viability was assessed in cells treated with DMSO or gossypol at different time intervals. (b) Cell viability was assessed in cells treated with DMSO or gossypol with various concentrations. Each experiment was repeated for three times. Data were presented as mean \pm SEM. $* P<0.05$ versus control.

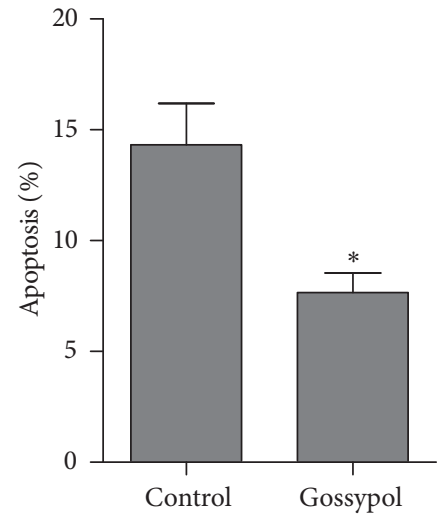

(a)

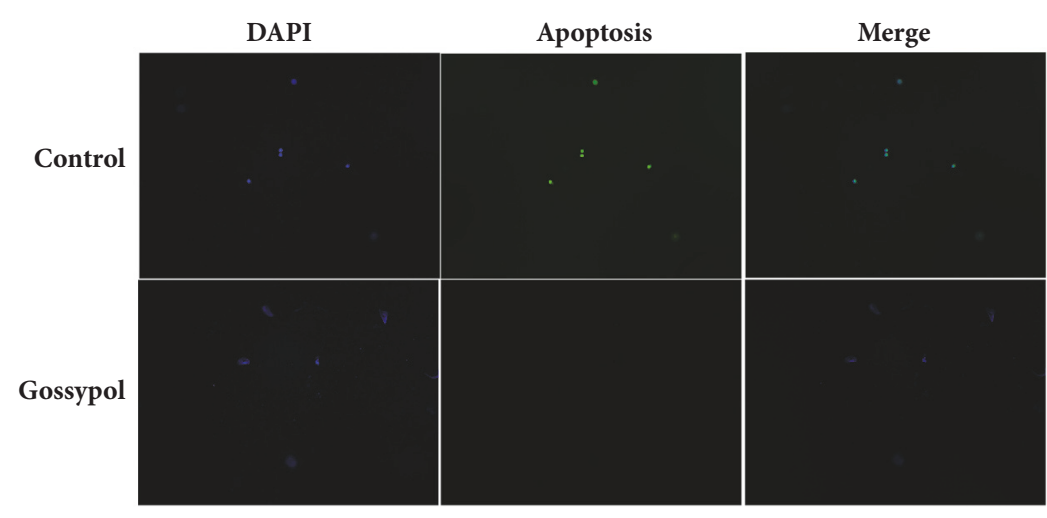

(b)

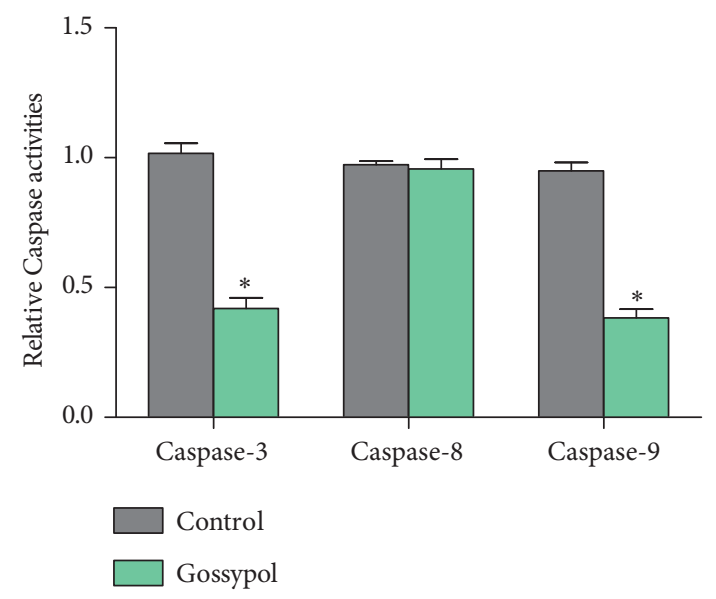

(c)

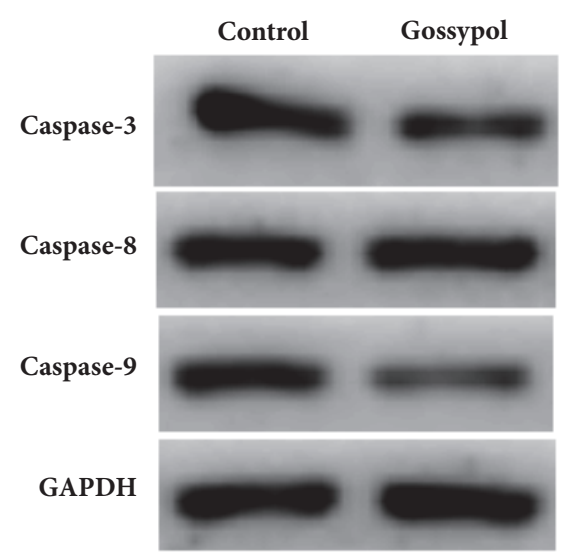

(d)

FIGURE 4: Gossypol inhibits cell apoptosis in osteoblast cells in vitro. (a) Cell apoptosis was determined in MC3T3-E1 cells treated with gossypol for $48 \mathrm{~h}$ with a concentration of $20 \mathrm{~nm}$. (b) TUNEL assay was performed to assess the cell apoptosis in cells treated with gossypol. Five random fields were selected for calculation. (c) Relative activities of caspase-3, caspase-8, and caspase- 9 in cells treated with gossypol. (d) Western blot assays were performed to examine the expression of caspase-3, caspase-8, and caspase-9 in MC3T3-E1 cells in response to gossypol treatments. Each experiment was repeated for three times. Data were presented as mean \pm SEM. $* P<0.05$ versus control. 


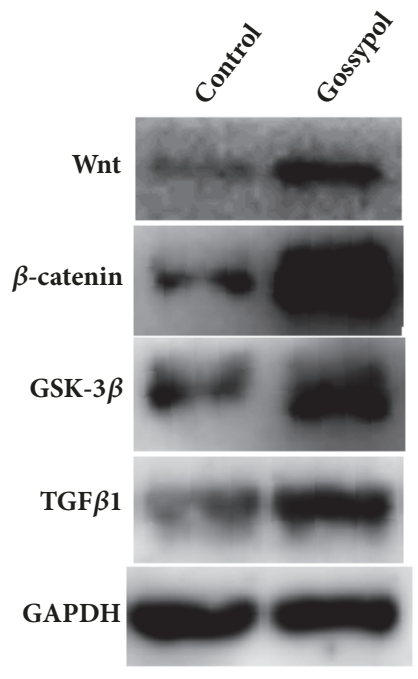

(a)

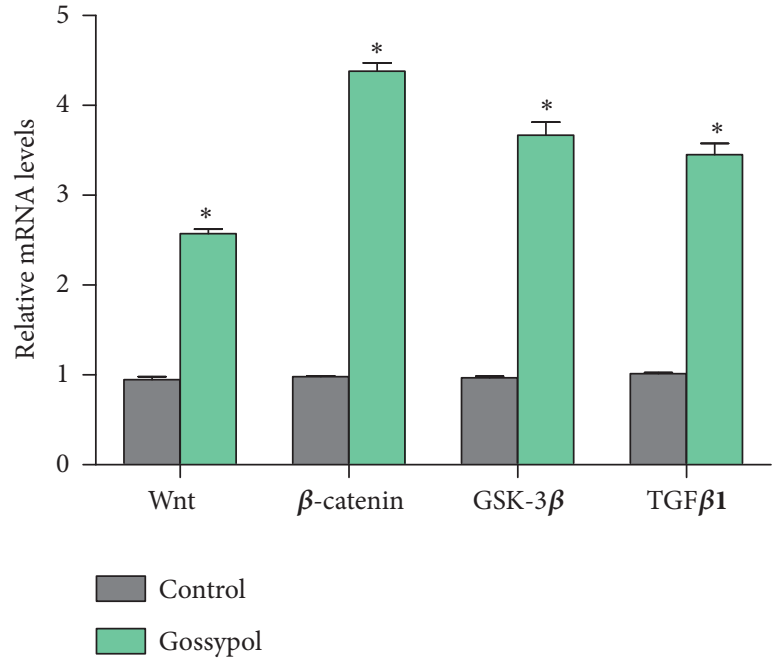

(b)

FIgURE 5: Gossypol treatments in MC3T3-E1 cells increases Wnt/ $\beta$-catenin signaling pathway. (a) Western blot assays examined the protein levels of Wnt, $\beta$-catenin, GSK-3 $\beta$, and TGF $\beta 1$ in cells treated with gossypol. (b) qRT-PCR assays examined the mRNA levels of Wnt, $\beta$-catenin, GSK- $3 \beta$, and TGF $\beta 1$ in cells treated with gossypol. Each experiment was repeated for three times. Data were presented as mean \pm SEM. $* P<0.05$ versus control.

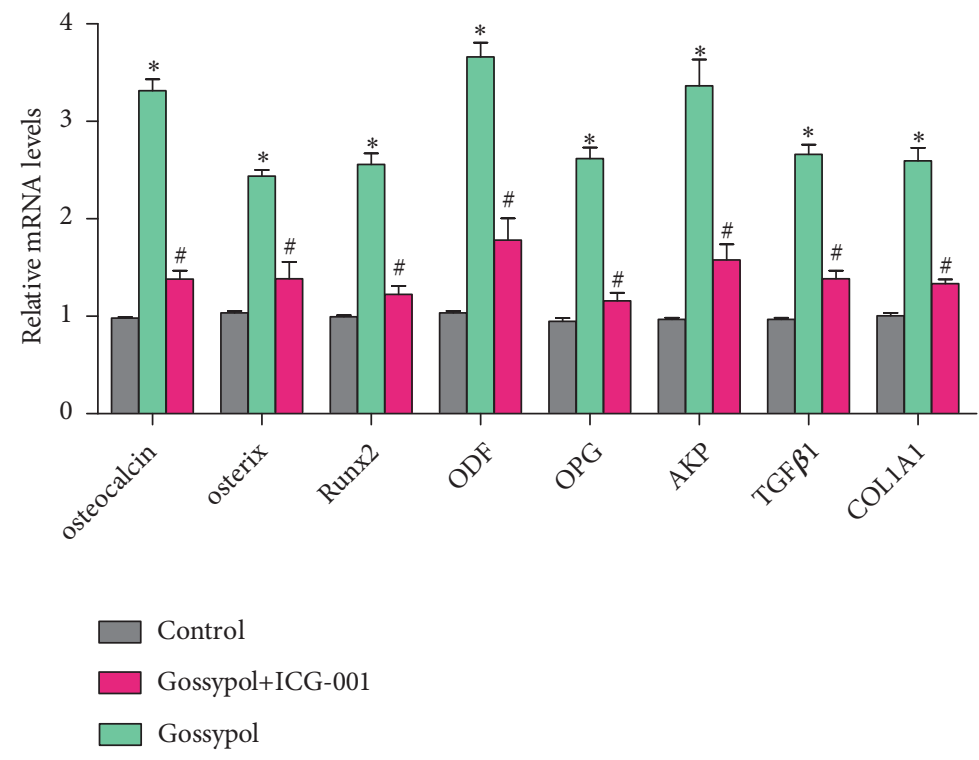

(a)

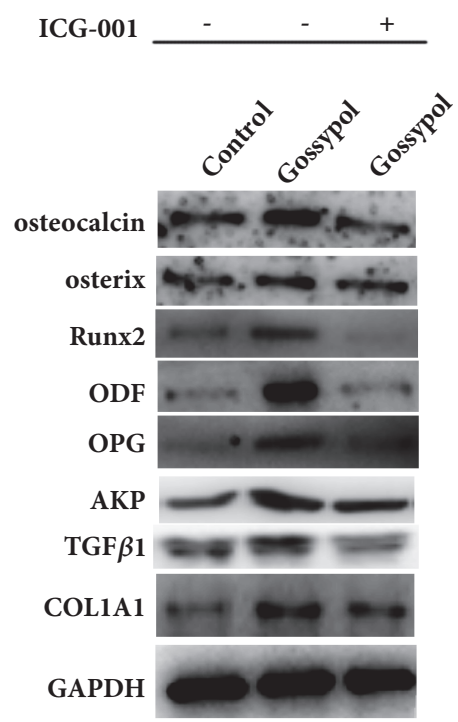

(b)

FIGURE 6: Inhibition of Wnt// $\beta$-catenin signaling pathway reversed the effects caused by gossypol in vitro. (a) mRNA levels of bone formation related genes in MC3T3-E1 cells treated with gossypol in presence or absence of ICG-001. $* P<0.05$, gossypol versus control. ${ }^{\#} P<0.05$, gossypol+ ICG-001 versus gossypol. (b) Protein levels of bone formation related genes (osteocalcin, osterix, Runx2, ODF, OPG, AKP, TGF $\beta 1$, and COL1A1) in MC3T3-E1 cells treated with gossypol in presence or absence of ICG-001. Each experiment was repeated for three times. Data were presented as mean \pm SEM. 
of bone turnover, causing higher bone resorption and lower bone formation, and eventually induces osteoporosis [2]. Promising drug therapies for ovariectomy-induced osteoporosis or senile osteoporosis have improved the living situations for the patients; however, certain drugs are not suitable for all patients and may cause some side effects for some individuals [18]. Thus, it is still necessary to discover novel drugs for the treatment of osteoporosis. Here, we showed that a new natural compound, gossypol, promoted bone formation in osteoporosis mice by inhibiting cell apoptosis via activating $\mathrm{Wnt} / \beta$-catenin pathway.

With the aid of microCT and histology methodology, it was initially found that gossypol significantly increased both cortical and medullar bone strength. The treatments of osteoporotic mice with gossypol also showed convincing turnover of osteoporosis as evidenced by the serum biochemical analysis. All these in vivo and in vitro data confirmed the beneficial roles of gossypol against osteoporosis. The activation of osteoblasts is regulated by multiple molecular signals, of which RANKL (receptor activator of nuclear factor kappa$B$ ligand) is the most important one. RANKL is produced by osteoblasts and lymphocytes and stimulates RANK. Osteoprotegerin (OPG) competes with RANK to bind to RNAKL, suppressing its ability to improve bone resorption [19]. Due to their important functions in osteoporosis, serum levels of osteocalcin, OPG, and RANKL were determined with Elisa kits and the results showed that gossypol treatment could promote bone formation through increasing their serum secretions. Therefore, it could be concluded that gossypol protected against osteoporosis in mice.

Cell apoptosis represents a critical biological process for disease development [20]. In this study, it was found that gossypol treatments decreased osteoblast cell viability. The inhibition of cell apoptosis by gossypol was mainly executed through activation of caspase-3 and caspase-9, two critical executors of intrinsic apoptotic caspases [21, 22]. Hence, gossypol promoted bone formation mainly through inhibiting osteoblast cell apoptosis.

Previous studies have demonstrated that Wnt/ $\beta$-catenin signaling is crucial for bone formation in human osteoporosis [23]. Activation of the Wnt/ $\beta$-catenin could increase trabecular and cortical bone mass through activation of mesenchymal stem cells, blockade of osteoblast apoptosis, and induction of osteoblast differentiation [24]. Furthermore, this pathway is an enticing target for drug development of osteoporosis and even other skeletal health problems [25]. Similar to those previous studies, we found that gossypol treatment in MC3T3-E1 cells increased Wnt/ $\beta$-catenin signaling and inhibition of this pathway with its specific inhibitor and ICG-001 reversed the protective effects of gossypol on bone formation. The regulation of osteoblast cell apoptosis by $\mathrm{Wnt} / \beta$-catenin signaling, therefore, might pave the way for gossypol-mediated bone formation.

\section{Conclusion}

Our study identified gossypol as a potential therapeutic drug for osteoporosis. Gossypol promoted bone formation by activating the $\mathrm{Wnt} / \beta$-catenin signaling and thereby decreasing cell apoptosis. These results provided novel insights into the treatment of osteoporosis in clinic.

\section{Data Availability}

The data used to support the findings of this study are available from the corresponding author upon request.

\section{Conflicts of Interest}

The authors declare that no conflicts of interest exist.

\section{Authors' Contributions}

Jinqian Liang and Chong Chen performed most of the experiments. Hongzhe Liu and Xiangyang Liu helped with the experiments and analyzed the data. Zheng Li helped with the reference and the manuscript. Hong Zhao and Jianhua $\mathrm{Hu}$ provided the funding, designed the study, and prepared the manuscript. Hong Zhao and Jianhua Hu contribute equally.

\section{Acknowledgments}

This study was sponsored by the Opening Project of People's Hospital of Hunan Province. We thank Dr. David from Johns Hopkins University for revising the manuscript for us.

\section{References}

[1] Share with women, "Osteoporosis," Journal of Midwifery \& Women's Health, vol. 48, no. 2, pp. 155-156, 2003.

[2] "Preventing Osteoporosis," Journal of Midwifery \& Women's Health, vol. 61, no. 2, pp. 289-290, 2016.

[3] Patient information from the Hormone Foundation, "Osteoporosis and women's health," The Journal of Clinical Endocrinology \& Metabolism, vol. 89, 2004.

[4] "Osteoporosis medications and your dental health," The Journal of the American Dental Association, vol. 142, no. 11, p. 1320, 2011.

[5] Information from your family doctor, “Osteoporosis," American Family Physician, vol. 69, pp. 1207-1208, 2004.

[6] For the dental patient, "Osteoporosis medications and oral health," The Journal of the American Dental Association, vol. 140, no. 6, p. 812, 2009.

[7] J. Paccou, P. Hardouin, A. Cotten, G. Penel, and B. Cortet, "The role of bone marrow fat in skeletal health: Usefulness and perspectives for clinicians," The Journal of Clinical Endocrinology \& Metabolism, vol. 100, no. 10, pp. 3613-3621, 2015.

[8] Summaries for patients, "Increasing the detection and treatment of osteoporosis in patients who present to an emergency department with a wrist fracture," Annals of Internal Medicine, vol. 141, no. 5, p. I54, 2004.

[9] E. Gielen, S. Boonen, D. Vanderschueren et al., "Calcium and Vitamin D Supplementation in Men," Journal of Osteoporosis, vol. 2011, Article ID 875249, 6 pages, 2011.

[10] P. R. Ebeling, R. M. Daly, D. A. Kerr, and M. G. Kimlin, "Building healthy bones throughout life: an evidence-informed strategy to prevent osteoporosis in Australia," Medical Journal of Australia, vol. 199, no. 7, p. S1, 2013. 
[11] G. A. Wells, A. Cranney, J. Peterson et al., "Alendronate for the primary and secondary prevention of osteoporotic fractures in postmenopausal women.", Cochrane Database of Systematic Reviews (Online), no. 1, p. CD001155, 2008.

[12] G. A. Wells, A. Cranney, J. Peterson et al., "Etidronate for the primary and secondary prevention of osteoporotic fractures in postmenopausal women," Cochrane Database of Systematic Reviews, p. D3376, 2008.

[13] D. Haguenauer, B. Shea, P. Tugwell, G. A. Wells, and V. Welch, "Fluoride for treating postmenopausal osteoporosis," Cochrane Database of Systematic Reviews, p. D2825, 2000.

[14] J. L. Judge, D. J. Nagel, K. M. Owens et al., "Prevention and treatment of bleomycin-induced pulmonary fibrosis with the lactate dehydrogenase inhibitor gossypol," PLoS ONE, vol. 13, no. 5, p. e0197936, 2018.

[15] N. Wen, Y. Dong, R. Song et al., "Zero-Order Release of Gossypol Improves Its Antifertility Effect and Reduces Its Side Effects Simultaneously," Biomacromolecules, vol. 19, no. 6, pp. 1918-1925, 2018.

[16] S. R. Saleh, R. Attia, and D. A. Ghareeb, "The Ameliorating Effect of Berberine-Rich Fraction against GossypolInduced Testicular Inflammation and Oxidative Stress," Oxidative Medicine and Cellular Longevity, vol. 2018, Article ID 1056173, 13 pages, 2018.

[17] C. Chen, W. Lin, K. Wong, K. Cheng, Y. Leung, and S. Yang, "Gossypol stimulates opening of a $\mathrm{Ca}(2+)$ - and $\mathrm{Na}(+)$-permeable but $\mathrm{Ni}(2+)$ - and $\mathrm{Co}(2+)$-impermeable pore in bEND. 3 endothelial cells," Clinical and Experimental Pharmacology and Physiology, vol. 45, no. 8, pp. 788-796, 2018.

[18] L. J. Dominguez, G. D. Bella, M. Belvedere, and M. Barbagallo, "Physiology of the aging bone and mechanisms of action of bisphosphonates," Biogerontology, vol. 12, no. 5, pp. 397-408, 2011.

[19] L. G. Raisz, "Pathogenesis of osteoporosis: concepts, conflicts, and prospects," The Journal of Clinical Investigation, vol. 115, no. 12, pp. 3318-3325, 2005.

[20] L. Yan, N. Guo, Y. Cao et al., "miRNA145 inhibits myocardial infarctioninduced apoptosis through autophagy via Akt3/ mTOR signaling pathway in vitro and in vivo," International Journal of Molecular Medicine, 2018.

[21] X. Zhong, L. Xiu, G. Wei et al., "Bezafibrate prevents palmitateinduced apoptosis in osteoblastic MC3T3-E1 cells through the NF- $\kappa \mathrm{B}$ signaling pathway," International Journal of Molecular Medicine, vol. 28, pp. 535-542, 2011.

[22] X. Zhu, X. Song, K. Xie, X. Zhang, W. He, and F. Liu, “Osthole induces apoptosis and suppresses proliferation via the PI3K/Akt pathway in intrahepatic cholangiocarcinoma," International Journal of Molecular Medicine, vol. 40, no. 4, pp. 1143-1151, 2017.

[23] V. V. Shanbhogue, K. Brixen, and S. Hansen, "Age- and SexRelated Changes in Bone Microarchitecture and Estimated Strength: A Three-Year Prospective Study Using HRpQCT," Journal of Bone and Mineral Research, vol. 31, no. 8, pp. 15411549, 2016.

[24] C.-L. Shen, I.-S. Kwun, S. Wang et al., "Functions and mechanisms of green tea catechins in regulating bone remodeling," Current Drug Targets, vol. 14, no. 13, pp. 1619-1630, 2013.

[25] J. Waalen, "Current and emerging therapies for the treatment of osteoporosis," Journal of Experimental Pharmacology, vol. 2, pp. 121-134, 2010. 


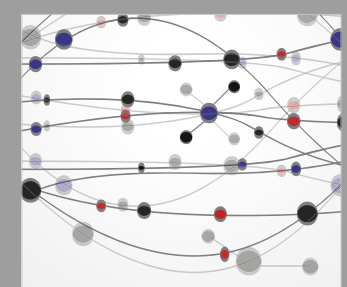

The Scientific World Journal
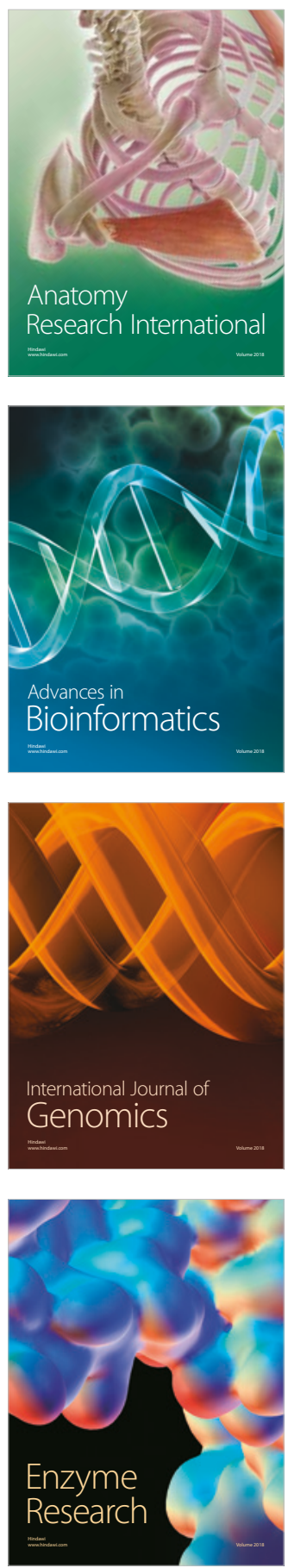
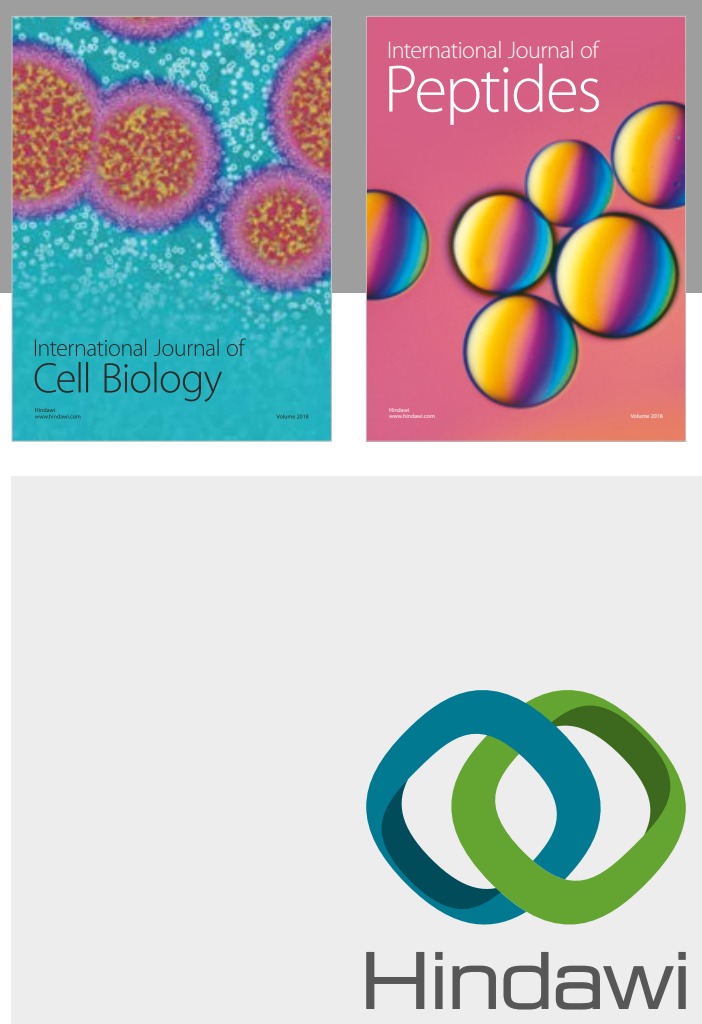

Submit your manuscripts at

www.hindawi.com
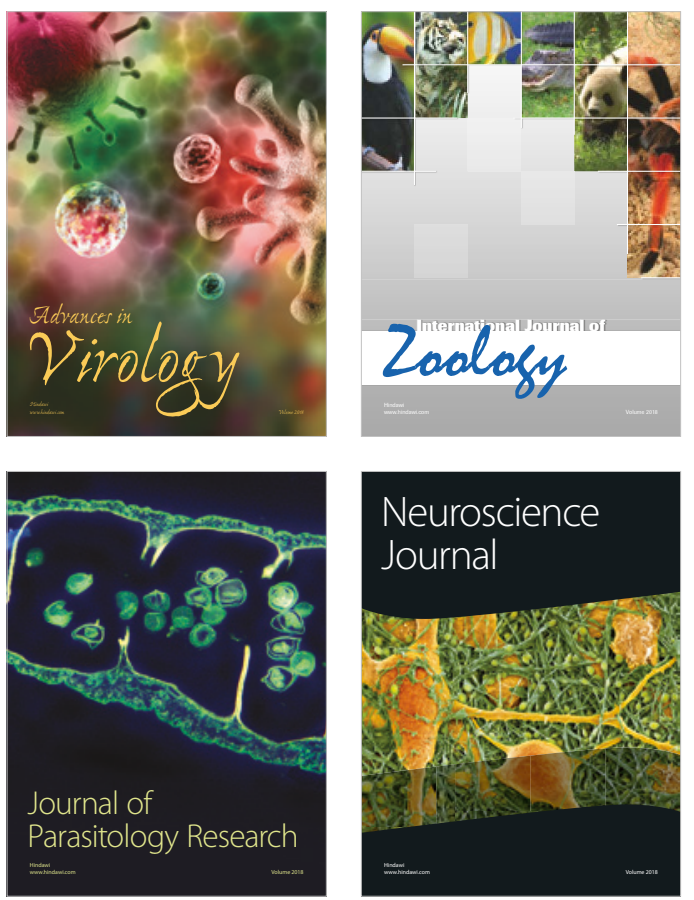
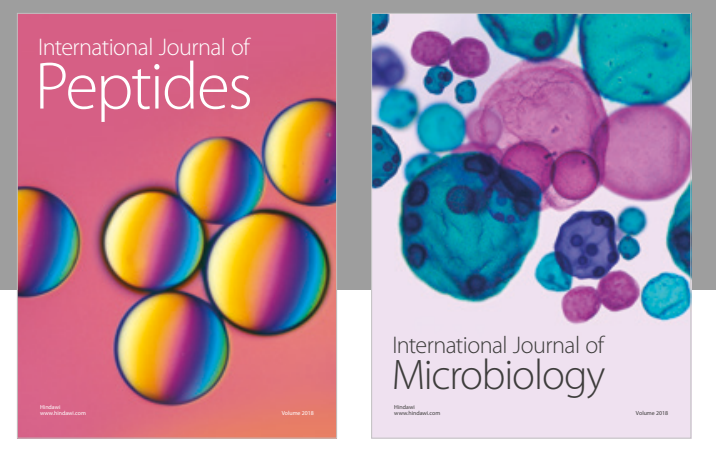

nternational Journal of Microbiology
Journal of
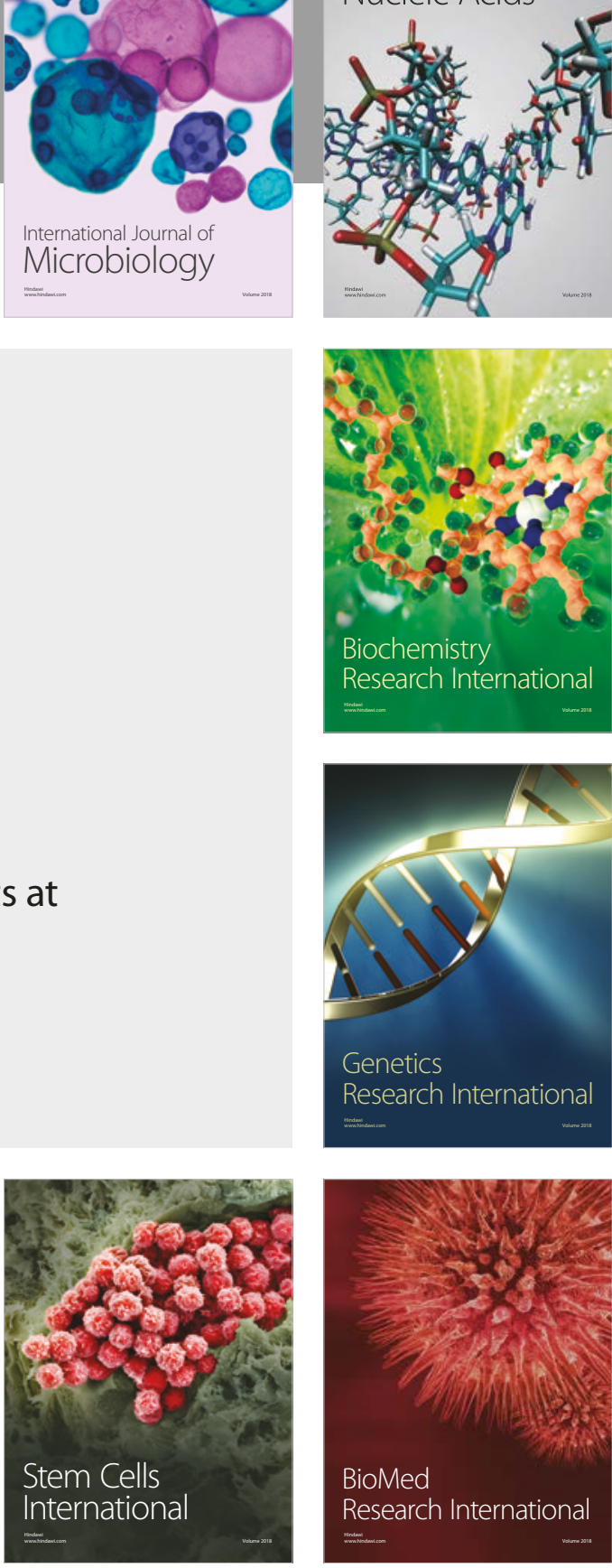
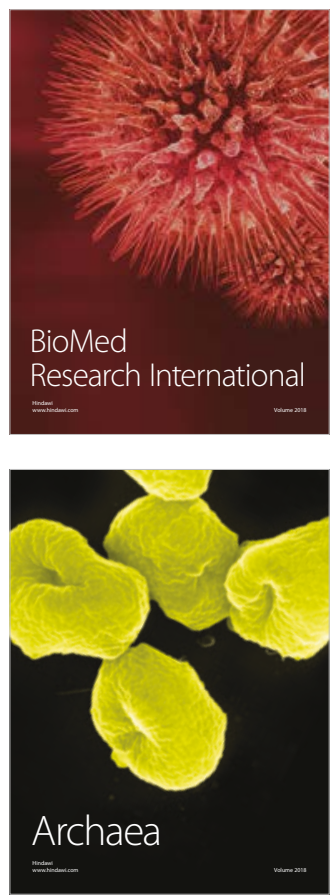